\title{
LRCH1 polymorphisms linked to delayed encephalopathy after acute carbon monoxide poisoning identified by GWAS analysis followed by Sequenom MassARRAY ${ }^{\circledast}$ validation
}

Jiapeng $\mathrm{Gu}^{1 \dagger}$, Jiao Zeng ${ }^{1+}, X_{i}$ Wang $^{1}$, Xin $\mathrm{Gu}^{1}$, Xiaoli Zhang ${ }^{1}$, Ping Zhang ${ }^{1}$, Fan Zhang ${ }^{1}$, Yongkai Han ${ }^{1}$, Yazhou Han ${ }^{2}$, Hongxing Zhang ${ }^{3}$, Wenqiang $\mathrm{Li}^{4^{*}}$ and Renjun $\mathrm{Gu}^{1^{*}}$ (D)

\begin{abstract}
Background: We explored the association of leucine-rich repeats and calponin homology domain containing 1 ( $(\mathrm{RCH} 1)$ gene polymorphisms with genetic susceptibility to delayed encephalopathy after acute carbon monoxide poisoning (DEACMP), which might provide a theoretical basis for the pathogenesis, diagnosis, and prognosis research of DEACMP.

Methods: Four single nucleotide polymorphisms, rs1539177 (G/A), rs17068697 (G/A), rs9534475 (A/C), and rs2236592 (T/C), of LRCH1, selected as candidate genes through genome-wide association analysis, were genotyped in 661 patients (DEACMP group: 235 cases; ACMP group: 426 cases) using Sequenom Massarray ${ }^{\oplus}$. The association analysis of four SNPs and LRCH1 was performed under different genetic models.

Results: LRCH1 polymorphisms (rs1539177, rs17068697, rs9534475) under additive and dominant genetic models were significantly associated with an increased risk of DEACMP, but no significant association under allele and recessive models was found. The LRCH1 rs2236592 polymorphism was susceptible to DEACMP only under the dominant model (TT/TC + CC, OR $=1.616,95 \% \mathrm{Cl}: 1.092-2.390, P=0.015784)$. In addition, the A allele gene of rs9534475 polymorphism in LRCH1 might increase the risk for DEACMP $(\mathrm{OR}=1.273,95 \% \mathrm{Cl}: 1.013-1.601, P=$ 0.038445).
\end{abstract}

Conclusions: We found a significant association between the four $\mathrm{LRCH} 1$ polymorphisms and DEACMP. The allelic A of rs9534475 polymorphism in LRCH1 might be a risk factor for DEACMP.

Keywords: DEACMP, ACMP, LRCH1 polymorphisms, SNP genotyping, Genetic model

\footnotetext{
*Correspondence: Iwq781603@163.com; gurenjun1961@163.com Jiapeng Gu and Jiao Zeng are Co-first authors

${ }^{4}$ International Joint Research Laboratory for Psychiatry and Neuroscience of Henan, Henan Key Lab of Biological Psychiatry of Xinxiang Medical

University, Xinxiang City 453002, Henan Province, China

${ }^{1}$ Henan Mental Hospital, The Second Affiliated Hospital of Xinxiang Medical University, No. 388 Jianshe Middle Road, Muye District, Xinxiang City 453002, Henan Province, China

Full list of author information is available at the end of the article
}

(c) The Author(s). 2019 Open Access This article is distributed under the terms of the Creative Commons Attribution 4.0 International License (http://creativecommons.org/licenses/by/4.0/), which permits unrestricted use, distribution, and reproduction in any medium, provided you give appropriate credit to the original author(s) and the source, provide a link to the Creative Commons license, and indicate if changes were made. The Creative Commons Public Domain Dedication waiver (http://creativecommons.org/publicdomain/zero/1.0/) applies to the data made available in this article, unless otherwise stated. 


\section{Background}

Carbon monoxide (CO) poisoning occurs after prolonged inhalation of $\mathrm{CO}$ at a lethal dosage of $100 \mathrm{ppm}$ or higher [1]. CO poisoning, as a leading gas poisoning event in the United States, affects 50,000 people per year [2]. Reportedly, CO poisoning events are more common in China than in western countries due to the large Chinese population base and usage of primary energy sources [3]. Delayed encephalopathy after acute carbon monoxide poisoning (DEACMP) is one of most severe complications of $\mathrm{CO}$ poisoning and occurs after a 2-60 day latency phase, in which the symptoms of acute $\mathrm{CO}$ poisoning transiently disappear [4]. The main clinical manifestations of DEACMP are mental disturbances, slow responses, cognitive dysfunction, gatism, dystonia, and even dementia [5]. So far, there is no specific therapy for DEACMP treatment; it has a poor prognosis with different degrees of intellectual disability and cognitive disorder as well as long-term agrypnocoma after treatment [6].

The specific pathogenesis of DEACMP has not yet been clarified. Generally recognized theories include the ischemia and hypoxia theory, the inflammation and immune injury theory, and the excitatory neurotransmitter theory [7]. Recently, accumulating evidence suggests that DEACMP is associated with a genetic factor. Populations with specific gene polymorphisms, such as LP-PLA2 rs1805017/ rs1051931, LRP1B rs1541976, and NRXN3 rs11845632/rs2196447, have genetic susceptibility to DEACMP [8-10]. To identify other related gene polymorphisms, a genome-wide association study (GWAS) was conducted in our previous study, which involved DNA samples from 175 DEACMP and 277 ACMP patients; allele and genotype frequencies of all detected single nucleotide polymorphisms (SNPs) were compared between those two groups [8]. Subsequently, four SNPs (rs1539177, rs17068697, rs9534475, and rs2236592) in the Leucine-rich repeats and calponin homology domain containing 1 (LRCH1) gene were identified as potential candidate sites associated with DEACMP (Additional file 1: Table S1).

LRCH1 belongs to a member of the LRCH family, which has a leucine-rich repeat domain and a calponin homology domain [11]. Little is currently known regarding the healthy biological function of this protein. Multiple SNP sites of LRCH1 are connected with osteoarthritis (OA) [12]. The pathogenesis of both OA and DEACMP is involved in immunization; hence, we speculate that LRCH1 may participate in the occurrence of DEACMP through an inflammatory immune mechanism. The latest research on LRCH1 by Xu and colleagues has provided more theoretical references for our conjecture [13]. They observed that LRCH1 acted as a novel DOCK8-interacting protein to inhibit $\mathrm{T}$ cell migration. $\mathrm{T}$ cells are closely related to the occurrence and development of various inflammatory diseases [14]. However, the association analysis between LRCH1 and DEACMP has not yet been reported. Collectively, we examined whether several LRCH1 SNPs influence the nosogenesis of DEACMP.

In recent years, the Sequenom Massarray ${ }^{\oplus}$ has proven to be an efficient method for genetic susceptibility gene detection screened by SNP genotyping [15]. Thus, in this study, the Sequenom method was used to further verify four candidate LRCH1 gene polymorphisms in DEACMP and ACMP patients with large samples. This study explored the associations of LRCH1 gene polymorphisms with genetic susceptibility to DEACMP, which might provide a theoretical basis for the pathogenesis, diagnosis, and prognosis research related to DEACMP.

\section{Methods}

\section{Subjects}

The study was approved by the Ethical Committee of the Second Affiliated Hospital of Xinxiang Medical University. The diagnostic criteria of DEACMP were as follows: (1) patients who had a history of coma due to acute $\mathrm{CO}$ poisoning within the last 2 months; (2) patients who experienced a latent phase (2-60 days) before the appearance of delayed symptoms; (3) delayed acute dementia of the central nervous system (CNS) damage as the primary clinical manifestation; and (4) EEG, CT, or MRI abnormalities. Meanwhile, the diagnostic criteria of "Occupational Acute Carbon Monoxide Poisoning" (GBZ23-2002) was used to diagnose ACMP disease. The ACMP patients experienced a history of coma due to acute $\mathrm{CO}$ poisoning, and no DEACMP event occurred following recovery for more than 90 days.

In addition to meeting the above diagnostic criteria, the subjects in the DEACMP and ACMP groups were both of Han Chinese descent from North Henan province and older than 40 years of age. Patients with other diagnosed nervous system dysfunctions, a history of infection within the previous 15 days, or recently administered hormone or immunosuppressive therapy were excluded.

In total, 661 patients (DEACMP group: 235 cases; ACMP group: 426 cases) were recruited from several hospitals in three cities during December 2017 to November 2006, including the First Affiliated Hospital of Xinxiang Medical University, the Second Affiliated Hospital of Xinxiang Medical University, the Third Affiliated Hospital of Xinxiang Medical University, Xinxiang Central Hospital, Xinxiang First People's Hospital, Xinxiang Second People's Hospital, the 371 Central Hospital of People's Liberation Army, Wuzhi County People's Hospital, Henan Grace Hospital, the People's Hospital of Anyang City, Qinyang People's Hospital, Jiaozuo Municipal Second Peoples' Hospital, and General Hospital of Ansteel Group. All participants provided written informed consent. 
The mean age, gender, and education levels of patients in the DEACMP and ACMP groups were matched. The peripheral blood samples from DEACMP patients were collected into anticoagulant vacuum tubes, which were collected from 6:00 am to 8:00 am following an overnight fast. Using the same method, the blood samples from ACMP patients were collected within $24 \mathrm{~h}$ of fully conscious recovery. All blood samples were labeled and stored at $-80^{\circ} \mathrm{C}$.

\section{Individual genotyping}

Genomic DNA from peripheral blood samples of each patient was extracted using a TIANamp Blood DNA Midi Kit (DP332, Tiangen Biotech, Beijing, China). The method for SNP genotyping of all samples was based on the Sequenom Massarray ${ }^{\circ}$ platform [16].

The design of all amplification primers of 4 SNPs of the LRCH1 gene was followed by gene sequencing using the dbSNP database and added to the 5' 10-mer tag (ACGTTGGATG), as shown in Table 1. The locus-specific PCR was amplified through multiple PCR amplification. The initial multiple PCR amplification was conducted using a Sequenom amplification kit. The cycling conditions were set as follows: beginning denaturation at $94{ }^{\circ} \mathrm{C}$ for 4 min; 45 cycles of $94{ }^{\circ} \mathrm{C}$ for $20 \mathrm{~s}, 56^{\circ} \mathrm{C}$ for $30 \mathrm{~s}$, and $72{ }^{\circ} \mathrm{C}$ for $60 \mathrm{~s}$, followed by $72^{\circ} \mathrm{C}$ for $5 \mathrm{~min}$.

Subsequently, SAP (shrimp alkaline phosphatase) treatment was performed to remove free dNTPs in the reaction system. In total, $2 \mu \mathrm{l}$ of SAP Mix containing $1.53 \mu \mathrm{l}$ water, $0.17 \mu \mathrm{l}$ SAP buffer (10x), and $0.3 \mu$ l SAP enzyme $(1.7 \mathrm{U} / \mu \mathrm{l})$ was added into the above PCR system and incubated at $37^{\circ} \mathrm{C}$ for $40 \mathrm{~min}$, followed by $85^{\circ} \mathrm{C}$ for $5 \mathrm{~min}$. After the SAP treatment, $2 \mu$ EXTEND mix, including $0.619 \mu \mathrm{l}$ water, $0.94 \mu \mathrm{l}$ Extend primer mix 1, $0.2 \mu \mathrm{l}$ iPLEX Buffer Plus, $0.2 \mu$ iPLEX terminator, and $0.041 \mu \mathrm{l}$ iPLEX enzyme, was added to conduct the single base extension reaction. The PCR procedure was set as $94^{\circ} \mathrm{C}$ for 30 s; 39 cycles of $94{ }^{\circ} \mathrm{C}$ for $5 \mathrm{~min}$ (step 1), $52{ }^{\circ} \mathrm{C}$ for $5 \mathrm{~s}$, and $80^{\circ} \mathrm{C}$ for $5 \mathrm{~s}$ (steps two and three were repeated four times for one cycle); and $72{ }^{\circ} \mathrm{C}$ for $3 \mathrm{~min}$. Afterward, resin purification was performed.
Finally, the PCR product was transferred into a Spectro CHIP (Sequenom) using a MassARRAY Nanodispenser RS1000 microarrayer (Capital Bio-Technology, Beijing, China). The genotypes and alleles were detected by MALDI-TOF mass spectrometry, and the results were analyzed using Sequenom Typer 4.0 software. With the analyzed results, the four SNPs were individually genotyped in all samples.

\section{Statistical analysis}

SPSS 20.0 software (SPSS, Inc., Chicago, IL, USA) was used to analyze the original data, and the Chi-square ( $x 2)$ goodness of fit test combined with the $x^{2}$ test was used to examine whether the distributions of genotypes in both groups conformed to the Hardy-Weinberg equilibrium law. The Karl Pearson $x^{2}$ test of the $2 \times 2$ tables and $2 \times 3$ contingency table with one degree of freedom was applied to assess the relationship between genetic model or allele gene and the DEACMP risk. Odds ratios (ORs) and corresponding 95\% confidence intervals (95\% CIs) were calculated for the four SNPs.

\section{Results \\ Clinical characteristics}

The four candidate SNPs (rs1539177, rs17068697, rs9534475, and rs2236592) of the LRCH1 gene were located in the intron region of chromosome 13 (Table 2). The demographic and clinical data regarding numbers, age, gender distribution, and educational level of patients in two groups are shown in Table 3. Among the 661 participants, a total of 658 (DEACMP: 232; ACMP: 426), 656 (DEACMP: 234; ACMP: 422), 661 (DEACMP: 235; ACMP: 426), and 659 (DEACMP: 235; ACMP: 424) patients were genotyped with rs1539177 (G/A), rs17068697 (G/A), rs9534475 (A/C), and rs2236592 (T/C) polymorphisms, respectively. In addition, there was no statistical difference of mean age, gender distribution, and educational level between two groups for rs1539177 (mean age: $P=0.1299$; gender distribution: $P=0.077$; educational level: $P=0.121$ ), rs17068697 (mean age: $P=0.1285$; gender distribution: $P=$ 0.073; educational level: $P=0.119$ ), rs9534475 (mean age:

Table 1 Primers for LRCH1 polymorphisms (rs1539177, rs17068697, rs9534475 and rs2236592)

\begin{tabular}{|c|c|c|}
\hline LRCH1 SNP & PCR amplification primer sequences $\left(5^{\prime}-3^{\prime}\right)$ & Single base extension primer sequence \\
\hline \multirow[t]{2}{*}{ LRCH1 (rs1539177) } & ACGTTGGATGCTCTTTCAGGCTCTAATTT & CAGACATAAATGATCAATATGACCC \\
\hline & ACGTTGGATGTGCCCCTCAAGGAGTGATT & CAGACATAAATGATCAATATGACCT \\
\hline \multirow[t]{2}{*}{ LRCH1 (rs17068697) } & ACGTTGGATGGGGTCCATGCTTATCTCTTC & GGCGTCTGCTAAGTAAATTGCGTATA \\
\hline & ACGTTGGATGCTGCACCCAGCATAAATAAC & GGCGTCTGCTAAGTAAATTGCGTATG \\
\hline \multirow[t]{2}{*}{ LRCH1 (rs9534475) } & ACGTTGGATGACTATAGTCACTTAGGCCCC & ctcGGCCCCATAGTCAGAC \\
\hline & ACGTTGGATGCCAGTGCTTAGACTCAATGC & ctcGGCCCCATAGTCAGAA \\
\hline \multirow[t]{2}{*}{ LRCH1 (rs2236592) } & ACGTTGGATGCGACTTGAGAGTTAATGGAG & AGAAAGAGGATGATTTAAGGTACA \\
\hline & ACGTTGGATGTGACTCCTCTCTGAGATTCC & AGAAAGAGGATGATTTAAGGTACG \\
\hline
\end{tabular}


Table 2 Physical locations of LRCH1 polymorphisms

\begin{tabular}{lllll}
\hline Variant & Chrom & Position & Annotation & MAF \\
\hline rs1539177 G/A & 13 & $46,725,920$ & intron & 0.38 (A) \\
rs17068697 A/G & 13 & $46,719,680$ & intron & $0.26(G)$ \\
rs9534475 A/C & 13 & $46,718,476$ & intron & 0.40 (C) \\
rs2236592 T/C & 13 & $46,721,781$ & intron & 0.44 (C) \\
\hline
\end{tabular}

MAF Minor allele frequency

$P=0.1285$; gender distribution: $P=0.071$; educational level: $P=0.123$ ), and rs2236592 (mean age: $P=0.1271$; gender distribution: $P=0.072$; educational level: $P=0.129$ ) (Table 3). Notably, the genotype distributions of these four SNPs between the two groups were within the Hardy-Weinberg equilibrium law (all $P>0.05$, Table 4).

\section{Association analysis between rs1539177, rs17068697,} rs9534475, and rs2236592 polymorphisms and DEACMP

The results of the association analysis between four SNPs under different genetic models and increased risk of DEACMP are presented in Table 5. The allele frequencies of rs1539177, rs17068697, and rs2236592 polymorphisms between patients in both groups were similar without significant differences (all $P>0.05$ ). Notably, there was no correlation between the rs1539177, rs17068697, rs9534475, and rs2236592 polymorphisms under the recessive model and increased risk of DEACMP $(P=0.50527, P=0.466963$, $P=0.742672$, and $P=0.61508$, respectively).

The association analysis between rs17068697 and DEACMP showed that rs17068697 was associated with susceptibility to DEACMP under the additive model (GG/ AG/AA, OR $=1.279,95 \%$ CI: $1.007-1.624, P=0.043729$ ) and the dominant model (GG/AG + AA, OR $=1.511,95 \%$ CI: $1.070-2.133, P=0.018579)$. Similarly, the rs17068697 was associated with susceptibility to DEACMP under the additive model (GG/AG/AA, OR $=1.285$, 95\% CI: $1.008-$ 1.6381, $P=0.043125)$ and the dominant model (GG/AG + AA, OR $=1.770,95 \% \mathrm{CI}: 1.147-2.729, P=0.009228)$.

The A allele frequency of rs 9534475 polymorphism was significantly higher than the $\mathrm{C}$ allele frequency in DEACMP patients $(\mathrm{OR}=1.273,95 \% \mathrm{CI}: 1.013-1.601, P=0.038445)$. In addition, the rs 9534475 polymorphism under the additive model (AA/AC/CC, OR $=1.285,95 \% \mathrm{CI}$ : 1.017-1.623, $P=0.035878)$ and dominant model $(\mathrm{AA} / \mathrm{AC}+\mathrm{CC}, \mathrm{OR}=$ 1.617, 95\% CI: $1.147-2.280, P=0.005935)$ were associated with an increased risk of DEACMP. Analysis of rs2236592 demonstrated that only rs2236592 polymorphism under the dominant model (TT/TC $+\mathrm{CC}, \mathrm{OR}=1.616,95 \%$ CI: $1.092-2.390, P=0.015784)$ increased susceptibility to DEACMP.

\section{Discussion}

We performed the genotype frequency analyses for the four LRCH1 polymorphisms (rs1539177, rs17068697, rs9534475, and rs2236592) in two groups and the association analysis of these four LRCH1 polymorphisms with the occurrence of DEACMP under different genetic models. The results revealed that the three $\mathrm{LRCH} 1$ polymorphisms (rs1539177, rs17068697, rs9534475) under additive and dominant genetic models were associated with an increased risk for DEACMP. The LRCH1 rs2236592 polymorphism was susceptible to DEACMP under the dominant model (TT/TC $+\mathrm{CC})$. In addition, the A allele gene of rs9534475 polymorphism might increase the risk of DEACMP.

Although the pathogenesis of DEACMP is complex and poorly revealed, mechanisms of immune injury-induced inflammatory reactions can cause the neurological damage associated with DEACMP $[17,18]$. Thom et al. have reported that DEACMP is immune-mediated and associated with chemically modified myelin basic protein (MBP) [19]. The degraded MBP becomes an autoantigen, which acts on macrophages and CD-4 lymphocytes to infiltrate the brain. Similarly, Xiang et al. have suggested that DEACMP is caused by MBP-level changes from acute $\mathrm{CO}$ intoxication with induced neutrophils and inflammatory response activation [20]. In addition, the elevated levels of IL-2, IL4 , IL-1 $\beta$, IL-6, IL-10, IL-8, and TNF- $\alpha$ in the cerebrospinal fluid of DEACMP patients are reported to be predictive markers of DEACMP [21-23]. Those studies have demonstrated that immune molecules mediated immunopathological damage plays a crucial role in the occurrence of DEACMP.

In our previous GWAS analysis, LRCH1 was identified as a candidate gene related to DEACMP [8], and in this study, the results of SNP genotyping revealed that four LRCH1 polymorphisms (rs1539177, rs17068697, rs9534475, and rs2236592) were closely related to LRCH1. LRCH1 is located in chromosome 13q14 and extensively expressed in vivo; however, the specific functions of its encoded proteins are still poorly understood. So far, increased studies have reported that $\mathrm{LRCH} 1$ gene polymorphisms are associated with osteoarthritis $[24,25]$. Spector et al. reported that an rs912428 polymorphism $(\mathrm{C} / \mathrm{T})$ in intron 1 of $\mathrm{LRCH} 1$ was linked with an increased risk of knee osteoarthritis via a possible structural role [24]. Additionally, Panoutsopoulou et al. found that another rs754106 polymorphism in intron 1 of LRCH1 was susceptible to osteoarthritis and the $\mathrm{T}$ allele gene increases risk of knee osteoarthritis by 1.14 times when compared with the $C$ allele gene [25]. It is widely known that dysregulated innate immune responses can result in osteoarthritis via mediation of chronic inflammation [26]. In addition, the immunologic changes of high levels of some cytokines (IL1, IL-10, TNF- $\alpha$, and IL-17) and high $\mathrm{CD}^{4+} / \mathrm{CD}^{+} \mathrm{T}$ cell ratio are detected in arthritis and osteoarthritis samples [27]. Considering that the pathogenesis of osteoarthritis and DEACMP are both involved in immunologic mechanisms, these findings imply that LRCH1 


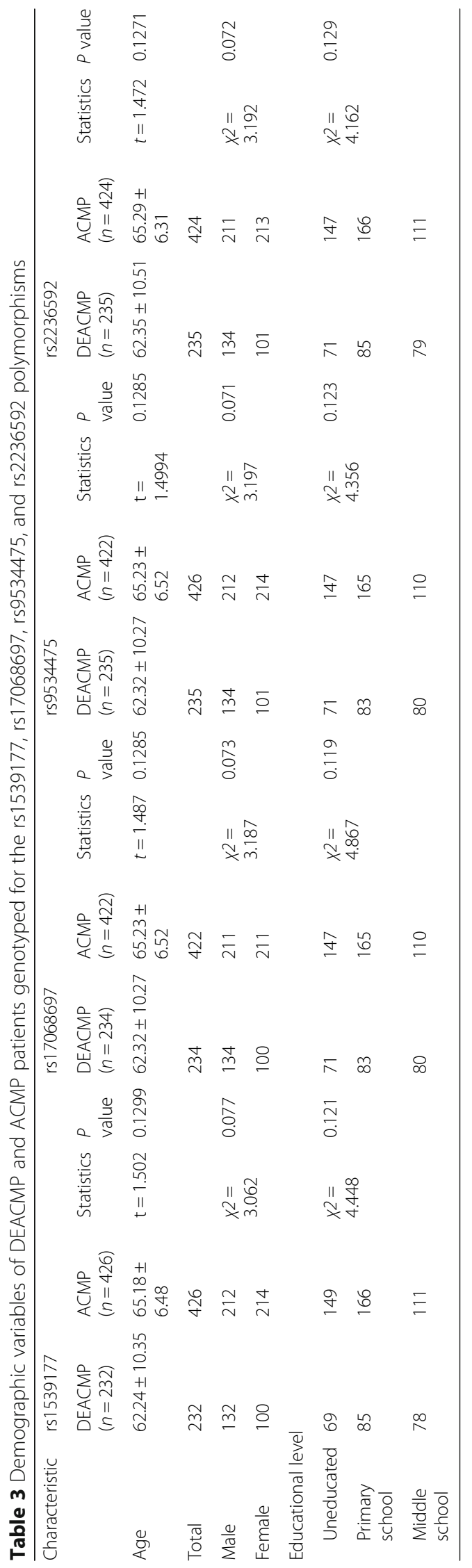


Table 4 Results of Hardy-Weinberg Equilibrium test for genotypes distributions of LRCH1 polymorphisms

\begin{tabular}{|c|c|c|c|c|c|}
\hline SNP & Genotypes & risk allele & Risk allele frequency ACMP/DEACMP & Actual value & Test value \\
\hline \multirow[t]{3}{*}{ rs1539177 } & GG & A & & 162 & $x 2=0.344340179 P=0.557334638$ \\
\hline & $A G$ & & $0.297 / 0.433$ & 206 & \\
\hline & AA & & & 58 & \\
\hline \multirow[t]{3}{*}{ rs17068697 } & GG & $A$ & & 95 & $X 2=2.145715207 P=0.142968447$ \\
\hline & $A G$ & & $0.5071 / 0.562$ & 226 & \\
\hline & AA & & & 101 & \\
\hline \multirow[t]{3}{*}{ rs9534475 } & AA & $C$ & & 167 & $X 2=0.196790791 P=0.657323794$ \\
\hline & $A C$ & & $0.378 / 0.4362$ & 196 & \\
\hline & $\mathrm{CC}$ & & & 63 & \\
\hline \multirow[t]{3}{*}{ rs2236592 } & $\pi$ & C & & 115 & $X 2=1.62565207 P=0.202305581$ \\
\hline & $\mathrm{TC}$ & & $0.4646 / 0.5149$ & 224 & \\
\hline & CC & & & 85 & \\
\hline
\end{tabular}

might also be associated with the occurrence of DEACMP through its participation in immunologic mechanisms.

Recently, LRCH1 is found to compete with Cdc42 for DOCK8 binding, which induces $\mathrm{T}$ cell migration and relieves autoimmune encephalomyelitis [13]. Similarly, $\mathrm{CD}^{+}{ }^{+} \mathrm{CD} 25^{+}$Treg and $\mathrm{CD} 8^{+} \mathrm{CD} 28^{-}$Treg infiltration into the CNS has been reported to play a crucial role in the pathogenesis of autoimmune encephalomyelitis via the regulation of local inflammation [28]. Notably, DEACMP is also a dysregulated CNS disease with autoimmune responses, which involve many of the immunocytes and cytokines mentioned above. Thus, these findings further supported our perspective that LRCH1 might also be associated with the occurrence of DEACMP via the regulation of $\mathrm{T}$ cell migration and migration into the CNS to mediate inflammation and immune reactions.

The associations of four LRCH1 polymorphisms and DEACMP are newly discovered. Our results showed that LRCH1 polymorphisms (rs1539177, rs17068697, and rs9534475) were susceptible to DEACMP under additive and dominant genetic models, indicating the risk alleles in rs1539177 (A), rs17068697 (A), and rs9534475 (C) might increase the occurrence of DEACMP based on additive and dominant autosomal inheritance patterns. The risk allele $\mathrm{C}$ in rs1539177 might also increase the risk of DEACMP based on the dominant autosomal inheritance pattern.

Table 5 Correlation analysis of LRCH1 polymorphisms under different genetic models and DEACMP risk

\begin{tabular}{|c|c|c|c|c|c|c|}
\hline \multirow{2}{*}{$\frac{\text { SNPs }}{\text { rs1539177 }}$} & \multicolumn{2}{|c|}{ Genetic models } & \multirow{2}{*}{$\begin{array}{l}\text { DEACMP } \\
263 / 201\end{array}$} & \multirow{2}{*}{$\frac{\text { ACMP }}{530 / 322}$} & \multirow{2}{*}{$\frac{P_{\text {obs }}}{0.050346}$} & \multirow{2}{*}{$\frac{\mathrm{OR}(95 \% \mathrm{Cl})}{0.795(0.632-1.001)}$} \\
\hline & allele & $(G / A)$ & & & & \\
\hline & additive & $(\mathrm{GG} / \mathrm{AG} / \mathrm{AA})$ & $67 / 129 / 36$ & $162 / 206 / 58$ & 0.043729 & $1.279(1.007-1.624)$ \\
\hline & dominant & $(G G / A G+A A)$ & $67 / 165$ & $162 / 264$ & 0.018579 & $1.511(1.070-2.133)$ \\
\hline & recessive & $(G G+A G / A A)$ & $196 / 36$ & $368 / 58$ & 0.50527 & $1.165(0.743-1.828)$ \\
\hline \multirow[t]{4}{*}{ rs17068697 } & allele & $(G / A)$ & $205 / 263$ & $416 / 428$ & 0.056602 & $0.802(0.639-1.006)$ \\
\hline & additive & $(G G / A G / A A)$ & $33 / 139 / 62$ & $95 / 226 / 101$ & 0.043125 & $1.285(1.008-1.6381)$ \\
\hline & dominant & $(G G / A G+A A)$ & $33 / 201$ & $95 / 327$ & 0.009228 & $1.770(1.147-2.729)$ \\
\hline & recessive & $(G G+A G / A A)$ & $172 / 62$ & $321 / 101$ & 0.466963 & $1.146(0.794-1.653)$ \\
\hline \multirow[t]{4}{*}{ rs9534475 } & allele & $(A / C)$ & $265 / 205$ & $530 / 322$ & 0.038445 & $1.273(1.013-1.601)$ \\
\hline & additive & $(\mathrm{AA} / \mathrm{AC} / \mathrm{CC})$ & $67 / 137 / 37$ & $167 / 196 / 63$ & 0.035878 & $1.285(1.017-1.623)$ \\
\hline & dominant & $(\mathrm{AA} / \mathrm{AC}+\mathrm{CC})$ & $67 / 168$ & $167 / 259$ & 0.005935 & $1.617(1.147-2.280)$ \\
\hline & recessive & $(\mathrm{AA}+\mathrm{AC} / \mathrm{CC})$ & $198 / 37$ & $363 / 63$ & 0.742672 & $1.077(0.693-1.674)$ \\
\hline \multirow[t]{4}{*}{ rs2236592 } & allele & $(\mathrm{T} / \mathrm{C})$ & $228 / 242$ & $454 / 394$ & 0.080373 & $1.223(0.976-1.533)$ \\
\hline & additive & (TT/TC/CC) & $44 / 140 / 51$ & $115 / 224 / 85$ & 0.064827 & $1.254(0.986-1.597)$ \\
\hline & dominant & $(\mathrm{TT} / \mathrm{TC}+\mathrm{CC})$ & $44 / 191$ & $115 / 309$ & 0.015784 & $1.616(1.092-2.390)$ \\
\hline & recessive & $(T T+T C / C C)$ & $184 / 51$ & $339 / 85$ & 0.61508 & $1.105(0.748-1.634)$ \\
\hline
\end{tabular}




\section{Conclusions}

In summary, our results revealed a significant association between four LRCH1 polymorphisms (rs1539177, rs17068697, rs9534475, and rs2236592) and DEACMP patients. The allelic A of rs9534475 polymorphism might be a risk factor for developing DEACMP. However, the results were limited to a small part of the Han Chinese population from North Henan province and should be explored in other populations in the future.

\section{Supplementary information}

Supplementary information accompanies this paper at https://doi.org/10. 1186/s12881-019-0931-7.

Additional file 1: Table S1. SNPS associated with DEACMP in the

pooling-based GWAS in both genders.

\section{Abbreviations}

95\% Cls: 95\% confidence intervals; CO: Carbon monoxide; DEACMP: Delayed encephalopathy after acute carbon monoxide poisoning; GWAS: Genomewide analysis study; LRCH1: Leucine-rich repeats and calponin homology domain containing 1; MBP: Myelin basic protein; ORs: Odds ratios; SAP: Shrimp alkaline phosphatase; SNPs: Single nucleotide polymorphisms

\section{Acknowledgments}

The authors thank the patients, their families and the physicians who helped us to take clinical data and blood samples.

\section{Authors' contributions}

$J G$ and JZ conceived and designed the research. XW, XG, and XZ collected the data and performed the analysis. PZ, FZ, and $Y K-H$ interpreted the data. $\mathrm{YH}$ and $\mathrm{HZ}$ performed the statistical analysis. WL and RG drafted and revised the manuscript. All authors read and approved the final manuscript.

\section{Funding}

This work was supported by the National Natural Science Foundation of China (No. 81141071, No. 81671319); the Training Plan for Excellent Young Teachers in Colleges and Universities of Henan (No. 2016GGJS-106); the Science and Technology Project of Xinxiang (No. CXGG17030); and the Support Project for the Disciplinary Group of Psychiatry and Neuroscience, Xinxiang Medical University. The funding bodies played no role in the design of the study and collection, analysis, and interpretation of data and in writing the manuscript.

\section{Availability of data and materials}

All data generated and/or analyzed during the current study are available in these links (https://www.ncbi.nlm.nih.gov/snp/rs1539177, https://www.ncbi. nlm.nih.gov/snp/rs17068697, https://www.ncbi.nlm.nih.gov/snp/rs9534475, https://www.ncbi.nlm.nih.gov/snp/rs2236592).

\section{Ethics approval and consent to participate}

This study was approved by the Ethics Committee of the Second Affiliated Hospital of Xinxiang Medical University. All the participants provided written informed consent.

\section{Consent for publication}

Not applicable.

\section{Competing interests}

The authors declare that they have no competing interests.

\section{Author details}

${ }^{1}$ Henan Mental Hospital, The Second Affiliated Hospital of Xinxiang Medical University, No. 388 Jianshe Middle Road, Muye District, Xinxiang City 453002, Henan Province, China. ${ }^{2}$ Qinyang People's Hospital, Jiaozuo City 454550 , Henan Province, China. ${ }^{3}$ The Psychology College of Xinxiang Medical University, Xinxiang City 453002, Henan Province, China. ${ }^{4}$ International Joint
Research Laboratory for Psychiatry and Neuroscience of Henan, Henan Key Lab of Biological Psychiatry of Xinxiang Medical University, Xinxiang City 453002, Henan Province, China.

Received: 17 March 2019 Accepted: 28 November 2019

Published online: 16 December 2019

\section{References}

1. Yoan N. Carbon Monoxide Poisoning. Am Fam Physician. 2012;60(5):186-94.

2. Rose JJ, Wang L, Xu Q, Mctiernan CF, Shiva S, Tejero J, Gladwin MT. Carbon monoxide poisoning: pathogenesis, management, and future directions of therapy. Am J Respir Crit Care Med. 2017;195(5):596.

3. Xue J, Sun Q, Wang Y, Gao G, Shi L, Yu T. Features of carbon monoxide poisoning in China. Iran J Public Health. 2013;42(10):1192-3.

4. Yanagiha K, Ishii K, Tamaoka A. Acetylcholinesterase inhibitor treatment alleviated cognitive impairment caused by delayed encephalopathy due to carbon monoxide poisoning: two case reports and a review of the literature. Medicine. 2017;96(8):e6125.

5. Ochi S, Abe M, Li C, Mori Y, Ishimaru T, Yoshino Y, Yamazaki K, Mori T, Fukuhara $\mathrm{R}$, Tanimukai $\mathrm{S}$. The nicotinic cholinergic system is affected in rats with delayed carbon monoxide encephalopathy. Neurosci Lett. 2014;569(4):33.

6. Turan Mi, Çayır A, Olgun H. Delayed encephalopathy after acute carbon monoxide poisoning. Chin J Clin Rehabil. 2006;41(1):217-8.

7. Hu H, Pan X, Wan Y, Zhang Q, Liang W. Factors affecting the prognosis of patients with delayed encephalopathy after acute carbon monoxide poisoning. Am J Emerg Med. 2011;29(3):261-4

8. Li W, Zhang Y, Gu R, Zhang P, Liang F, Gu J, Zhang X, Zhang H, Zhang H. DNA Pooling Base genome-wide association study identifies variants at NRXN3 associated with delayed encephalopathy after acute carbon monoxide poisoning. PLoS One. 2013;8(11):e79159.

9. Yu J, Wang Y, Pan X. Association of CDH17 and LRP1B gene polymorphisms with delayed encephalopathy after carbon monoxide poisoning. Chin J Pract Nerv Dis. 2017;20(12):18-22.

10. Zhao N, Zhuo X, Guo B, Chenglei S, Yan X, Jing Yan SH, Tie X, Chen Y. LpPLA2 variants associated with delayed encephalopathy after acute carbon monoxide poisoning. Int J Clin Exp Med. 2016;9(8):16393-8.

11. Foussard H, Ferrer P, Valenti P, Polesello C, Carreno S, Payre F. LRCH proteins: a novel family of cytoskeletal regulators. PLoS One. 2010; 5(8):e12257.

12. Panoutsopoulou K, Thiagarajah S, Day-Williams A, Southam L, Doherty M, Zeggini $\mathrm{E}$, Wilkinson J. Improved phenotype definitions of $\mathrm{OA}$ in genetic association studies highlight a structural role for a common variant in LRCH1. Osteoarthr Cartil. 2015;23:A70.

13. Xu X, Han L, Zhao G, Xue S, Gao Y, Xiao J, Zhang S, Chen P, Wu Z-y, Ding J. $\mathrm{LRCH} 1$ interferes with DOCK8-Cdc42-induced T cell migration and ameliorates experimental autoimmune encephalomyelitis. J Exp Med. 2017; 214(1):209-26

14. O'Connell RM, Kahn D, Gibson WS, Round JL, Scholz RL, Chaudhuri AA, Kahn ME, Rao DS, Baltimore D. MicroRNA-155 promotes autoimmune inflammation by enhancing inflammatory T cell development. Immunity. 2010:33(4):607-19.

15. Smith HM, Smith BP, Morales NB, Moskwa S, Clingeleffer PR, Thomas MR. SNP markers tightly linked to root knot nematode resistance in grapevine (Vitis cinerea) identified by a genotyping-by-sequencing approach followed by Sequenom MassARRAY validation. PLoS One. 2018;13(2):e0193121.

16. Gabriel S, Ziaugra L, Tabbaa D. SNP genotyping using the Sequenom MassARRAY iPLEX platform. Curr Protoc Hum Gen. 2009;60(1):2-12.

17. Chen BD, Jing-Lun LI, Neurology DO. Progress of pathogenesis of delayed encephalopathy after acute carbon monoxide poisoning. Pract J Card Cereb Pneumal Vasc Dis. 2014;22(4):1-4

18. Ide T, Kamijo Y. The early elevation of interleukin 6 concentration in cerebrospinal fluid and delayed encephalopathy of carbon monoxide poisoning. Am J Emerg Med. 2009;27(8):992-6.

19. Thom SR, Bhopale VD, Zhang J, Gimotty P. From the cover: delayed neuropathology after carbon monoxide poisoning is immune-mediated. Proc Natl Acad Sci U S A. 2004;101(37):13660-5.

20. Xiang WP, Xue H, Wang BJ. Delayed encephalopathy of acute carbon monoxide intoxication in rats: potential mechanism and intervention of dexamethasone. Pak J Pharm Sci. 2014;27(6 Suppl):2025. 
21. Gu RJ, Wang XH, Zhang P, Lu H, Guo XS, Song JG, Zhang F. Change of the serum interleukin 6 in patients with delayed encephalopathy after acute carbon monoxide poisoning. Chin J Ind Hyg Occup Dis. 2005;23(6):461-2.

22. Zhen L, Gu RJ, Zhang P. Serum levels and clinical significance of IL in patients with delayed encephalopathy after acute carbon monoxide poisoning. Chin J Ind Hyg Occup Dis. 2008;26(9):561-2.

23. Zhang P, Ren-Jun GU. Changes of serum interleukin levels and its clinic significance in patients with delayed encephalopathy after acute carbon monoxide poisoning. J ClinNeurol. 2007;20(3):220-1.

24. Spector TD, Reneland RH, Mah S, Valdes AM, Hart DJ, Kammerer S, Langdown M, Hoyal CR, Atienza J, Doherty M. Association between a variation in LRCH1 and knee osteoarthritis: a genome-wide singlenucleotide polymorphism association study using DNA pooling. Arthritis Rheum. 2006;54(2):524-32.

25. Panoutsopoulou K, Thiagarajah S, Day-Williams AG, Southam L, Doherty M, Zeggini E, Wilkinson J. Improved phenotype definitions of OA in genetic association studies highlight a structural role for a common variant in LRCH1. Osteoarthr Cartil. 2015;23(2):A70.

26. Kalaitzoglou E, Humphrey MB. Dysregulated innate immune responses mediate chronic inflammation leading to osteoarthritis. In: Meeting of the American-Society-For-Bone-And-Mineral-Research, vol. 2014; 2014. p. S25.

27. Hussein MR, Fathi NA, El-Din AME, Hassan HI, Abdullah F, Al-Hakeem E, Backer EA. Alterations of the CD4 +, CD8 + T Cell Subsets, Interleukins-1 $\beta$, IL-10, IL-17, Tumor Necrosis Factor-a and Soluble Intercellular Adhesion Molecule-1 in Rheumatoid Arthritis and Osteoarthritis: Preliminary Observations. Pathol Oncol Res. 2008;14(3):321-8.

28. Shang XF, Xi NN, Wang T, LV J, Han Z, Zheng RY. The change of periphery and centra CD4(+);CD25(+);Treg, CD8(+);CD28(-);Treg in the MOG induced model of experimental autoimmune encephalomyelitis. Chin J Cell Mol Immunol. 2010;26(8):746-9.

\section{Publisher's Note}

Springer Nature remains neutral with regard to jurisdictional claims in published maps and institutional affiliations.

Ready to submit your research? Choose BMC and benefit from:

- fast, convenient online submission

- thorough peer review by experienced researchers in your field

- rapid publication on acceptance

- support for research data, including large and complex data types

- gold Open Access which fosters wider collaboration and increased citations

- maximum visibility for your research: over $100 \mathrm{M}$ website views per year

At $\mathrm{BMC}$, research is always in progress.

Learn more biomedcentral.com/submissions 